\title{
Physics of Amphiphiles, Micelles and Microemulsions
}

\author{
Vittorio Degiorgio, Pavia
}

(Dipartimento di Elettronica, Università di Pavia)

Advanced research in condensedmatter physics and statistical physics was, until recent times, confined to systems of simple molecules forming welldefined simple phases. However, most of the molecules and states of matter we meet in everyday life or find in biological systems or use in industrial processes are very complex, and their understanding requires specific experiments and new concepts. In the last twenty years, physicists have become more and more interested in such fields as polymers and biological macromolecules, liquid crystals, gels, membranes and, more recentIy, micelles and microemulsions. All these areas of research are now truly interdisciplinary, and show a very stimulating intermingling of fundamental and applied aspects.

The word amphiphile describes the presence in the same molecule of both hydrophilic and hydrophobic parts. The hydrophilic part (sometimes called the head of the amphiphile) can be charged or polar; the hydrophobic groups are usually hydrocarbons consisting of one or two linear chains. It is well known that when a hydrocarbon is in contact with water the network of hydrogen bonds between water molecules reconstructs itself to avoid the region occupied by hydrocarbon. This constraint on the local structure of water decreases the entropy of the water near the hydrocarbon and results in a larger free energy for the total system. The hydrophobic effect, therefore, arises because of the self-attraction of water for itself, which tends to squeeze the hydrocarbon out, and not because of the repulsion between water and hydrocarbon.

The opposition of properties within an amphiphilic molecule gives rise to the formation of monolayers at air-water interfaces. Since the partition coefficient between surface and bulk may be very large in aqueous amphiphile solutions, extremely low amphiphile concentrations may reduce very effectively the surface tension. For this reason amphiphiles are often called surfactants or surface-active compounds.

Amphiphile solutions present many challenging problems. Aggregation is a multiple equilibrium process controlled by intermolecular and interaggregate forces. The choice among all the possible shapes (globular, rodlike, or disclike micelle, vesicle) is primarily determined by geometric packing constraints, but the transition from one shape to the other may be induced by changing the temperature or concentration or by adding a third component. Furthermore, because of the existence of attractive intermicellar interactions, micellar solutions and microemulsions may present critical consolute points which strongly influence the properties of the system over a wide temperature (and concentration) region. The interparticle interactions may generate a large variety of structural phases, including liquid crystals, gels and viscoelastic solutions.

Over recent years research on amphiphile solutions has flourished. The extensive application of light and neutron scattering and of nuclear magnetic resonance has produced a wealth of important experimental results. Moreover, the utilization of advanced statistical-mechanics methods is leading to significant theoretical contributions.

\section{Micellar Properties}

The most remarkable property of amphiphiles in aqueous solution is that they self-associate to form structures in which the hydrophobic tails are in the middle, avoiding contact with water as much as possible, and the hydrophilic groups are at the surface. The simplest possible aggregate is a globular micelle, formed by $20-100$ monomers which assemble their hydrophobic tails in a hydrocarbon core and expose the polar (or ionic) heads to water (Fig. 1). An obvious geometric constraint to the shape of amphiphile aggregates is that any point inside the hydrophobic core cannot have a distance from the surface larger than a monomer length (usually $\cong$ $1.5-2.5 \mathrm{~nm}$ ). This means that the growth of micelles is restricted to one (rod) or two dimensions (disc). Some amphiphiles, like biological phospholipids, may form aggregates, called vesicles, consisting of a spherically concentric bilayer (or several).

From the point of view of applications, the most important property of micelles

Fig. 1 - Various amphiphile structures in aqueous solution, from J.N. Israelachvili, Ref. 4.
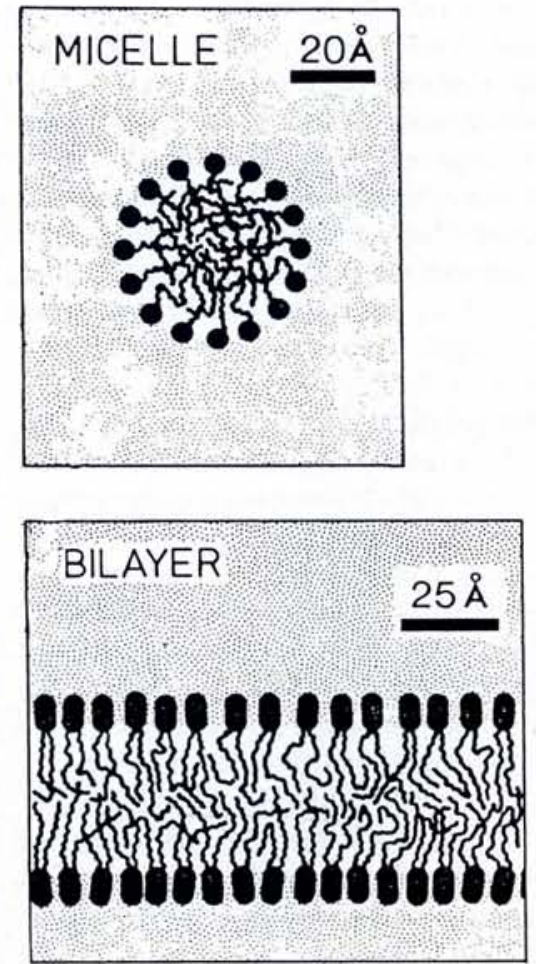
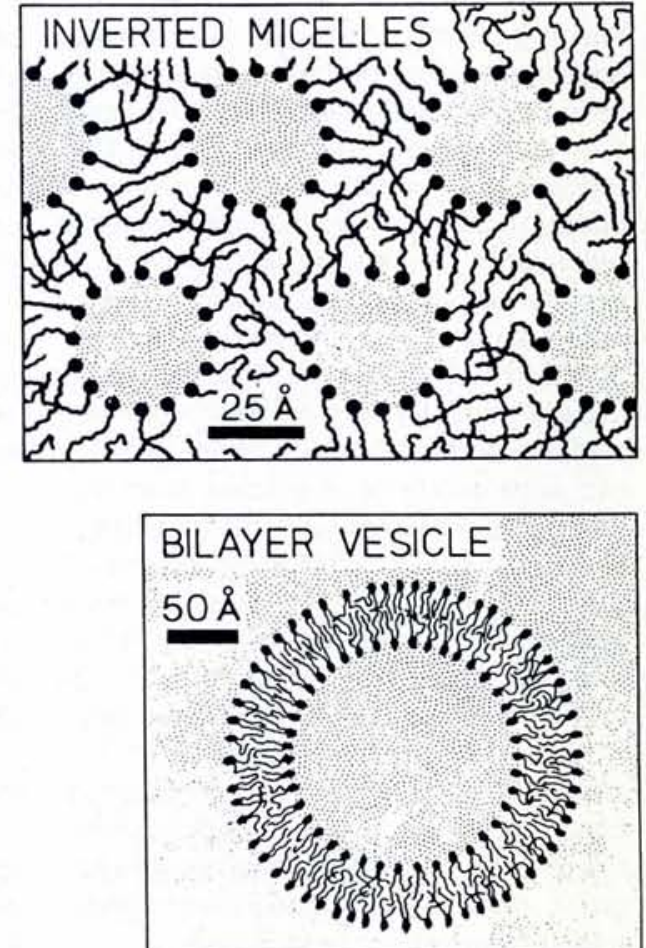


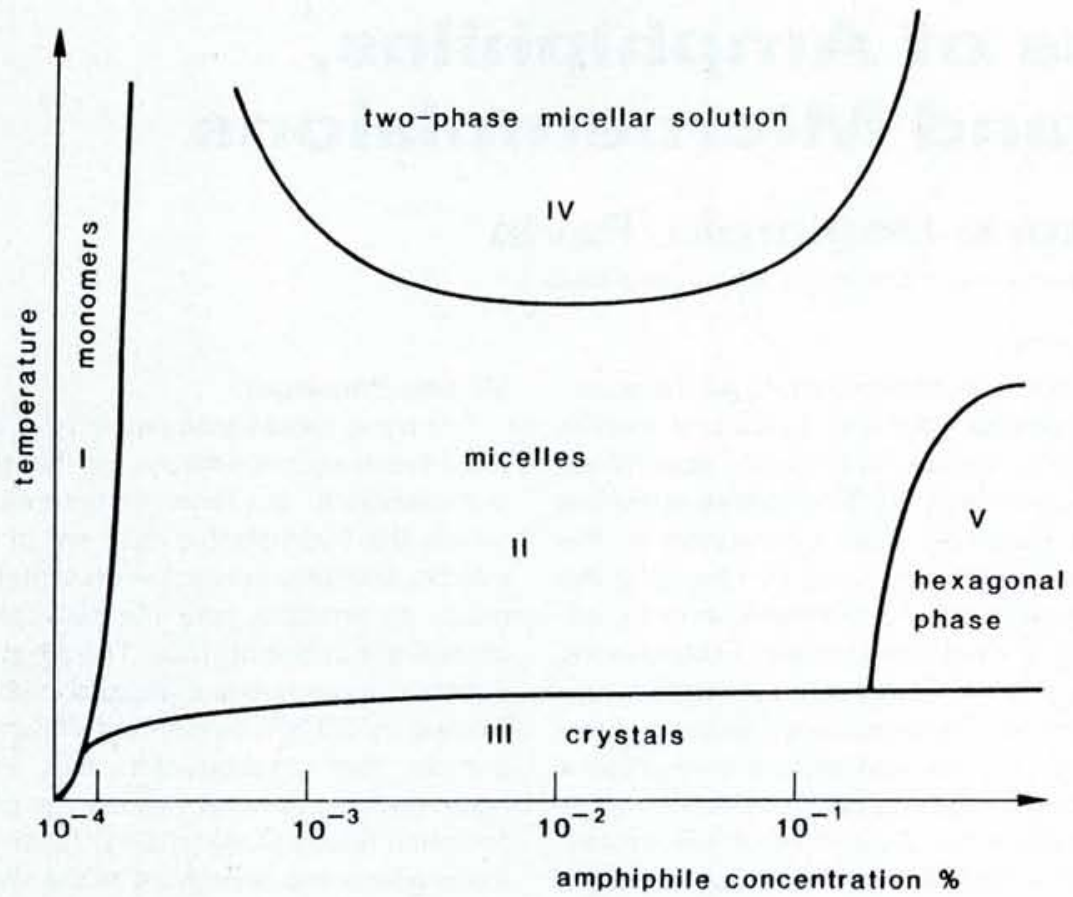

Fig. 2 - Typical phase diagram of a water-amphiphile system.

is that it is possible to solubilize within the hydrocarbon core, compounds which are insoluble or sparingly soluble in water. Clearly, this is relevant to many industrial (detergents, cosmetics, emulsion polymerization) and biological (cholesterol solubilization) processes. Vesicles are useful as membrane models, and may be helpful as drug carriers (drugs are solubilized in the inner water region).

In order to understand how the size and shape of the aggregate are chosen in a specific amphiphile solution, it is essential to realize that the hydrophilic headgroups do not completely cover the micellar surface, but leave an energetically unfavourable interface between the hydrocarbon core and water. The tendency to reduce this interface is counterbalanced by the electrostatic repulsion of the head groups, so that one can define an optimal surface area $a$ which an amphiphile molecule requires at the micellar surface. This area $a$ is usually much larger than the cross-section of a surfactant chain. From geometric considerations it follows that the parameter determining the aggregate shape $p=v / a l$, where $v$ is the volume of the surfactant chain. For $p \leq 1 / 3$ only globular micelles exist, for $1 / 3<p \leq 1 / 2$ rodlike micelles are formed, for $1 / 2 \leq p$ $<1$ disclike micelles may exist and for $p$ $=1$ an infinite bilayer is obtained. By changing temperature or amphiphile concentration, or by adding electrolytes, it is possible to modify the optimal headgroup area $a$, and thus to induce transitions in the aggregate structure.
When an amphiphile is dissolved in water, micelle formation occurs only if the temperature $T$ is above a critical value $T_{0}$ (critical micelle temperature) and the amphiphile concentration $c$ is above $c_{0}$ (critical micelle concentration). A qualitative phase diagram is shown in Fig. 2. Region I corresponds to an aqueous solution of monomeric amphiphile. In this region the amphiphile concentration is too low thermodynamically to favour micelle formation which implies the association of typically 100 monomers and is a highly co-operative process. Micelle formation is not a true phase separation process, so that, strictly speaking, there is not a critical concentration, but a narrow concentration range below which no micelles exist and above which virtually all added amphiphile enters the micellar state. Region II corresponds to an aqueous solution of micelles in dynamic equilibrium with monomers. The monomer concentration $c_{1}$ is in this region $\cong c_{0}$. The micelles are globular at low concentration, but they often become rodlike as the concentration is increased much above $c_{0}$. Region III corresponds to an aqueous solution of monomers co-existing with precipitated hydrated solid amphiphile. It is believed that at the phase boundary between regions II and III a conformational change of the hydrocarbon chain of the amphiphile occurs: for $T>T_{0^{\prime}}$ the chain is flexible, whereas, for $T<$ $T_{0}$, the chain is rigid. Micelles will not form below $T_{0}$, because rigid molecules will not easily pack into a micellar structure.
In region $\mathrm{V}$ the system is in a liquidcrystalline phase, the hexagonal phase, consisting of rod-shaped micelles of indefinite length packed in a hexagonal array and separated by a continuous water region. Other liquid-crystalline phases (lamellar, cubic,...) may appear in different concentration-temperature regions. A very interesting feature of amphiphile solutions is that lyotropic nematic phases have been found in some systems. These phases consist of aligned rod-or disc-like micelles, and can usually be oriented in magnetic fields. Region IV is a two-phase region, typical of nonionic micellar solutions, in which both phases are micellar. The behaviour of the system near the consolute curve is discussed below.

\section{Interacting Brownian Particles: Critical Phenomena}

The most direct way of determining the aggregate size and shape is by using light- or neutron-scattering techniques. However, the information obtained through a scattering experiment is usually a combination of single-particle properties and collective properties. If an appropriate model for the pair-interaction potential $V(r)$ is available, one can derive from the experiment not only $\mathrm{mi}$ cellar parameters, but also the relevant parameters describing $V(r)$.

Since micellar solutions may show a wide variety of sizes and shapes and of interaction potential, they represent a very good testing ground for theoretical calculations (or numerical simulations) on systems of interacting Brownian particles. Some recent experiments serve to illustrate this aspect.

Fig. 3 shows the collective diffusion coefficient $D$ of sodium dodecyl sulfate (SDS) micelles in aqueous solutions at various $\mathrm{NaCl}$ concentrations, as measured by quasielastic light scattering. The SDS micelles have an aggregation number around 100, and an electric charge about $40 \mathrm{e}$. Their shape is globular, with a hydrodynamic radius of $25 \mathrm{~nm}$. The dependence of $D$ on the micelle concentration $c$ can be explained, at least at $\mathrm{NaCl}$ concentrations below $0.5 \mathrm{~mol}$, by assuming that the micelle size and shape does not depend appreciably on $c$ and that the $c$-dependence of $D$ is only due to intermicellar interactions. For dilute solutions,

$$
D=D_{0}\left[1+k_{D}\left(c-c_{0}\right)\right],
$$

where $D_{0}$ is the translational diffusion coefficient of the SDS micelle and $k_{D}$ is a "dynamic second virial coefficient" related to the interparticle interaction potential $V(r)$ by an integral expression which 


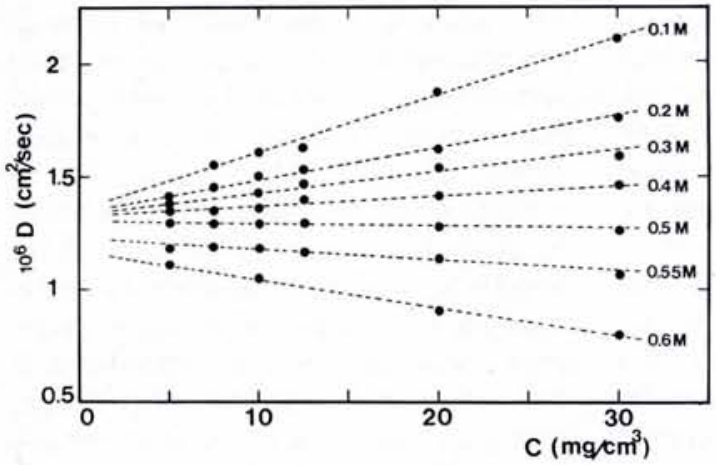

Fig. 3 - Quasielastic light scattering measurement of the diffusion coefficient in an ionic micellar solution as a function of the amphiphile concentration at various ionic strengths. concentration in preparation for the system comprising the rodlike structures of the hexagonal phase. The full lines in Fig. 4 represent theoretical calculations of the scattered neutron intensity performed by assuming that the micelles are hard spheres and by using the mean spherical approximation. When the temperature is increased, it is found that the fit to the experimental data requires the introduction of an attractive potential among the non-ionic micelles. This fin-

contains also hydrodynamic effects (because the movement of one particle through the fluid generates a velocity field which affects the motion of neighbouring particles). At low ionic strength $V(r)$ is dominated by the electrostatic repulsion term. At increasing ionic strength, the Coulomb potential is screened more and more effectively by the free ions, and both the excluded volume and attractive interaction become important. The fit to the experimental data allows one to determine the micellar radius (through $D_{\mathrm{o}}$ ) and the relevant parameters describing $V(r)$ (through $k_{D}$ ). It should be added that at high ionic strength (above $0.5 \mathrm{~mol} \mathrm{NaCl}$ ) micelles grow into elongated structures. Presently it is not clear whether such a growth can be described by an independent particle model or whether interactions play an essential role.

Fig. 4 shows small-angle neutron scattering results obtained with an aqueous solution of non-ionic micelles. Such micelles behave at room temperature and low concentration like hard spheres. The interesting feature of Fig. 4 is that the micelles remain globular even at concentrations which are close to the boundary between the isotropic micellar phase and the liquid-crystalline hexagonal phase. This behaviour is in contrast to the one found with ionic micelles which show a progressive growth with

Fig. 4-Scattered neutron intensity from a non-ionic micellar solution as a function of the modulus of the scattering vector at various amphiphile concentrations, from $M$. Corti et al., Chem. Phys. Lett. 109 (1984) 579.

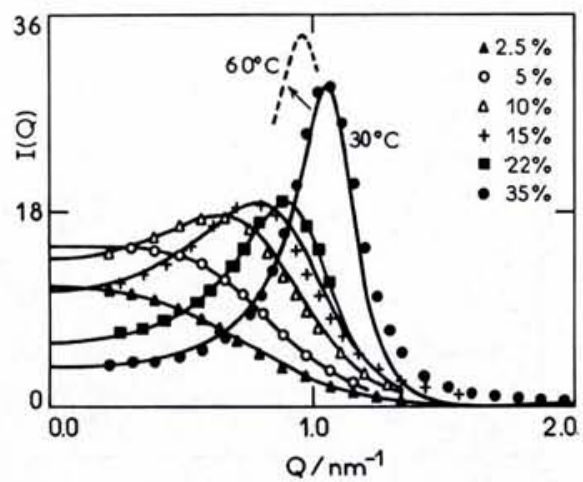

\section{Experimental
Nuclear Physicists}

There are vacancies for experimental physicists to join the Nuclear Structure Division at Daresbury Laboratory, an establishment of the Science and Engineering Research Counci which operates major national facilities for scientific research.

The group is involved in carrying out and supporting a research programme on the Nuclear Structure Facility (NSF), a large tandem accelerator which is operating at up to $20 \mathrm{MV}$ on terminal.

\section{Senior Scientific Officer}

\section{Ref. DL/911}

The successful applicant will be required to organise and direct work necessary for the operation, maintenance and development of major equipment for charged particle identification and detection used in programmes aimed at studies of nuclear collision dynamics, spectroscopy, and nuclei far from stability. Candidates will be expected to carry out research in conjunction with university users on the NSF and to play an active role in initiating and developing new programmes. Other duties will involve the overall coordination and scheduling of the scientific programme and the assessment of future needs for instrumentation and other facilities on the NSF.

Applicants (male or female) should have a good honours degree (or equivalent qualification) in physics and several years postgraduate experience in experimental nuclear research. A Ph.D qualification in nuclear physics and a period of relevant post-doctoral experience would be an advantage.

\section{Higher Scientific Officer}

\section{Ref. DL/913}

The successful applicant will be required to liaise with university research teams using the NSF. Candidates will also be expected to collaborate in nuclear research programmes and to play an active role in initiating and developing new programmes. Other duties will involve work on the design and development of major equipment through to commissioning, operation and maintenance. As a member of the in-house team of scientists he/she will be expected to provide expertise in the methods of experimental nuclear physics to aid and direct the technical and scientific support staff in operating the NSF and for diagnosing problems as they arise.

Applicants (male or female) should have a good honours degree (or equivalent qualification) in an appropriate discipline with a period of relevant post-graduate experience. $\mathrm{A} \mathrm{Ph.D}$ degree in nuclear physics and a period of post-doctoral experience in experimental nuclear research would be an advantage.

The salary scales according to qualifications and experience for the Higher Scientific Officer and Senior Scientific Officer posts are $£ 7,435$ to $£ 10,039$ per annum and $£ 9,329$ to $£ 12,050$ per annum respectively. These salary scales are currently under review.

There is a non-contributory superannuation scheme, a generous leave allowance and a flexible working hours scheme.

CLOSING DATE: 30 th June 1985.

For further information please write or telephone

Dr J. S. Lilley on Warrington (0925) 65000, Ext. 558.

Application forms may be obtained from and should be retumed quoting the appropriate reference number to: The Personnel Officer, Science \& Engineering Research Council, Daresbury, Warrington, WA4 4AD. 


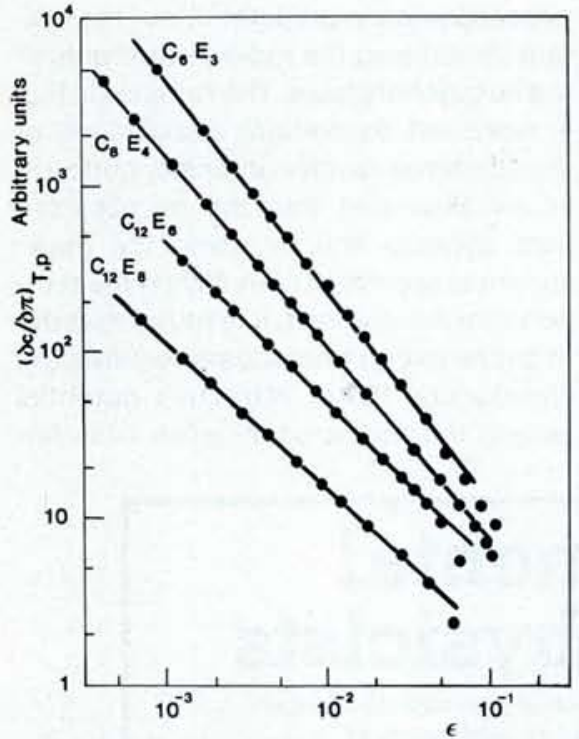

Fig. 5 - Light scattering measurement of the osmotic compressibility of non-ionic micellar solutions as a function of the temperature distance, $\varepsilon=\left(T_{c}-T\right) / T_{c}$, from the critical point. The slope of the plots represents the critical exponent $\gamma$ which appears to depend on the nature of the amphiphile.

ding is consistent with the existence of a consolute curve at high temperature (see Fig. 2) with a lower consolute point (critical point). The critical behaviour of non-ionic micellar solutions has been much investigated in recent years for two main reasons:

i) since the micelle is a spontaneous aggregate it may change size and shape on approaching the critical point, thus introducing a new degree of freedom in the critical behaviour,

ii) the observed critical exponents $\gamma$ (see Fig. 5) and $v$ do not coincide with those derived from the renormalization group calculation and measured for all critical fluids and critical binary mixtures. The origin of this departure from the law of universality of critical phenomena is presently unknown.

\section{Microemulsions}

So far I have discussed binary systems (amphiphile plus water) or pseudobinary systems (amphiphile plus salty water). A new phenomenology arises when we add to the amphiphile solution a water-insoluble compound (oil). Under appropriate selection of the amphiphile and of the oil, it is possible to prepare a clear, isotropic, non-viscous, thermodynamically stable phase containing large amounts of both water and oil. This phase is called a microemulsion. The simplest possible structure of a microemulsion consists of water (oil) droplets (typically, the radius is $100 \AA$ ) coated with an amphiphile layer, immersed in a sea of oil (water). The droplet structure may have a limited stability range in con- centration (and in temperature) because the radius of curvature of the interface between the amphiphile layer and the continuous phase is fixed by the nature of the amphiphile monomer and cannot be shifted too much from the optimum value. For instance, if we start with a water-in-oil microemulsion and we add water, the droplet should swell to accommodate the extra water, but the new droplet may now be unstable, because of the unfavourable bending energy, and the system may undergo phase separation by segregating a water-rich phase. In order to modify the bending energy of the interface, thus increasing the stability range of the microemulsion, an alcohol (typically butanol oil-amphiphile system.

It is important to note that the microemulsion structure does not always consist of droplets. In certain cases, as for instance in those region of the phase diagram near the transition between water-in-oil and oil-in-water droplets, the structure of the system seems to be better described by a bicontinuous random partition of oil and water which are separated by a fluctuating amphiphilealcohol interface.

The experimental study of microemulsion structure has been undertaken with a variety of scattering and NMR techniques. As an example, in Fig. 6 are shown some results obtained with self-diffusion NMR. It is clearly seen that state 3 corresponds to oil in water droplets (the diffusion coefficient $D$ of oil [toluene] and amphiphile [SDS] molecules is the same and is much lower than the diffusion coefficient of water) and that state 5 corresponds to water in oil droplets. In the intermediate cases the system does not show a well-defined droplet structure. Note also that $D$ is always low for the amphiphile, which is constrained to stay at interfaces, and takes an intermeor pentanol) is often added to the water-

diate value for butanol because some of the alcohol molecules are intercalated with SDS molecules at the interface and some move freely in the continuous phase.

Microemulsions present a large variety of phenomena of great interest for statistical physics. The microemulsion droplets may show an attractive pairpotential and exhibit critical phenomena characteristic of a gas-liquid transition, similar to the non-ionic micelles already discussed. Water-in-oil microemulsions show, at increasing droplet concentration, an enhancement of the electric conductivity which strongly suggest the existence of a percolation threshold. Furthermore, the interfacial tension between two coexisting microemulsion phases may be as low as $10^{-3}$ dyne/cm (five orders of magnitude smaller than the water-oil interfacial tension). At present, the understanding of the connections between all these phenomena is rather limited.

It is almost needless to say that microemulsions can be extremely important for applications. Most of the early work was aimed at increasing the efficiency of oil extraction from oil wells. Indeed, there are many situations in which a considerable fraction of the oil remains trapped in the microporosities of the well and can be recovered only by promoting, through additives, the formation of stable phases containing both water and oil.

\section{SUGGESTED READING}

1. Tanford C., The Hydrophobic Effect (Wiley) 1980.

2. Micellization, Solubilization, and Microemulsions, Ed. K.L. Mittal (Plenum Press) 1977.

3. Surfactants in Solution, Eds. K.L. Mittal, and B. Lindman (Plenum Press) 1984.

4. Physics of Amphiphiles Micelles, Vesicles and Microemulsions, Eds. V. Degiorgio and M. Corti (North-Holland) 1985.

Fig. 6 - NMR measurement of self-diffusion coefficients in a SDS-butanol-toluene-water microemulsion, from B. Lindman et al., J. Colloid Interface Sci. 83 (1981) 569.
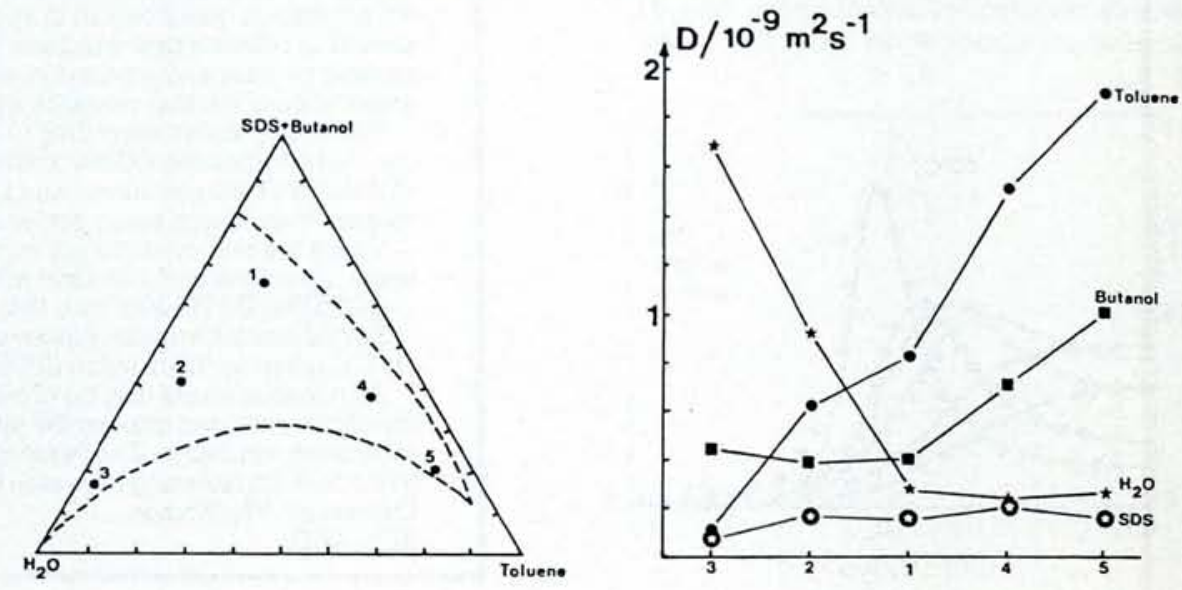